\title{
An Analytical Model for Studies of Soil Modification Effects on Ground Penetrating Radar
}

\author{
Jatupum Jenwatanavet and Joel T. Johnson, Member, IEEE
}

\begin{abstract}
Due to the similar dielectric constants of buried nonmetallic targets and dry soils, it is often difficult to detect and identify nonmetallic targets with ground penetrating radar. The addition of properly chosen chemical agents to modify soil properties can potentially provide improved detection. Previous studies using waveguide experiments have shown that the addition of water improves dielectric contrasts but also increases loss so that target detectability is not necessarily improved. The addition of liquid nitrogen to wet soils can reduce background medium loss and restore target visibility, and waveguide studies of target detection through controlled depth of nitrogen penetration have shown that scattering can be significantly enhanced if an optimal amount of nitrogen is added. In this paper, a simple physical optics (PO) model for scattering from a three-dimensional target buried below a half space is presented, and it is shown that the radar cross section of the target depends on the dielectric contrast with and attenuation in the background medium. The model is validated through comparison with a Method of Moments code and found to yield accurate predictions for near normal incidence geometries. Analytical studies of target detection with two concepts of soil modification are then described: obtaining an "optimal" homogeneous soil water content and the addition of a large quantity of water along with an optimal amount of liquid nitrogen. Finally, initial tests of these soil modification techniques with a dielectric rod antenna ground penetrating radar are performed and demonstrate that the addition of liquid nitrogen to excessively wet soils can reduce loss and enhance target visibility.
\end{abstract}

Index Terms-Ground penetrating radar, landmine detection, radar cross section, subsurface sensing.

\section{INTRODUCTION}

$\mathbf{T}$ HE detection and identification of nonmetallic anti-personnel landmines remains a challenging problem for all current technologies [1]. Ground penetrating radar (GPR) systems are currently only of limited utility in this area because of the often low dielectric (i.e., complex permittivity) contrast between plastic mines and the surrounding soil. For example, the relative permittivity $\epsilon_{r}$ of most plastic materials has a very small imaginary part and a real part in the range of $2-4$, while that of most dry soils is similar, making it difficult for a sensor which relies on scattering from dielectric contrasts to distinguish these two materials. Previous S-band waveguide studies [2] have shown that the homogeneous addition of water to dry sand can increase target/background medium dielectric

Manuscript received October 8, 1999; revised December 11, 2000. This work was supported by NSF under Project ECS-9701678 and by a grant from Duke University as part of the Office of the Secretary of Defense MURI on Humanitarian Deminig.

The authors are with the Department of Electrical Engineering, ElectroScience Laboratory, Ohio State University, Columbus, OH 43212 USA.

Publisher Item Identifier S 0018-926X(01)05251-6. contrasts so that larger scattered fields can be obtained. However, attenuation in the background medium is also increased and reduces scattered fields. For homogeneous water content soils an optimal water content exists to provide maximum target visibility, but for deeper targets even this "maximum" response can be quite small due to attenuation. For the nonhomogeneous water content soils created when water is poured onto a soil surface and allowed to penetrate over a moderate time period, previous waveguide experiments [3] have shown that moderate depth target visibility is not improved because a substantial quantity of water is required to obtain penetration to target depths and results in excessive losses near the surface for GPR systems operating at microwave frequencies. Thus the addition of water alone may not be sufficient to enhance target detection in many environments. Although many soil and target physical parameters can also influence GPR detection of buried objects [4], this study focuses on the influence of dielectric contrast on microwave frequency GPR systems for detection of small nonmagnetic, nonmetallic objects buried at relatively shallow depths (such as anti-personnel mines).

To address problems with excessive loss in wet soils, the addition of chemicals to the soil medium has been proposed [2], [3]. Although such a use of chemicals will clearly have many important practical issues which must be resolved before use in field tests is possible, the electromagnetic effects must first be considered in order to determine appropriate chemical choices. One chemical which has been proposed for modifying soil properties is liquid nitrogen [2], [3], which potentially could be generated on site for use in the field. Due to the much smaller loss tangents in ice than in water [5], target visibility can potentially be regained in excessively lossy soil situations. However, the dielectric constant of frozen soil also becomes more similar to that of nonmetallic targets, so again small dielectric contrasts become a problem in completely frozen soils. Waveguide experimental results [3] suggested that an "optimal" amount of liquid nitrogen should exist to enhance target visibility; this amount of nitrogen would be sufficient to freeze most of the soil above the target so that propagation loss is reduced, but would leave a small layer of wet soil around the target to retain dielectric contrast so that large scattered fields could be obtained. The importance of flow effects for water and liquid nitrogen have also been discussed [3]; improvements in target visibility in waveguide experiments were determined by subtracting measurements made with and without a surrogate mine target. Differences in the flow patterns of water and liquid nitrogen with and without a target can also be measured, but careful control of these chemicals was exercised to obtain repeatable measurements. Further waveguide analytical studies of soil modification through the addition of 
water and liquid nitrogen [6] suggested a procedure for locating low-contrast targets at unknown depths through a gradual addition of increasing amounts of liquid nitrogen. Waveguide experiments confirmed this concept, even with the complication of water and liquid nitrogen flow effects not considered in the analytical studies. The resulting procedure allows unknown depth targets which have exactly the same dielectric constant as the original soil medium to be detected in principle.

In this paper, previous results are extended beyond the waveguide configuration through an analytical study of the physical optics approximation for scattering from a three dimensional target buried below a half space. Although many numerical codes [7]-[12] have been developed for this problem, the analytical equations obtained in this paper allow insight into the effects of soil modification. Results show that the radar cross section (RCS) of a buried target is proportional to the reflection coefficient between the target and the surrounding medium, confirming the importance of dielectric contrast. The dependence on background medium attenuation and transmission through the soil/air interface is also clarified. The model is validated by comparison with a method of moments (MoM) code and found to yield reasonable predictions for near normal incidence angles. Analytical studies of target detection with two concepts of soil modification are also described: adding water to obtain an "optimal" homogeneous soil water content and the addition of a large quantity of water along with an optimal amount of liquid nitrogen. Results from the analytical study of the optimal homogeneous soil water content show that optimal water contents decrease when target depths increase since loss even in the lower water content soils is significant for very deep targets. The analytical study of the addition of a large quantity of water along with an optimal amount of liquid nitrogen demonstrates the potential of liquid nitrogen to reduce loss and restore target responses. Finally, the soil modification techniques suggested by these analytical studies are applied in an initial measurement with a dielectric rod 2-6-GHz GPR system developed at The Ohio State University ElectroScience Laboratory [13], and results suggest that the techniques proposed should be applicable to general microwave GPR systems for detecting nonmetallic anti-personnel mines.

\section{Physical Optics Model FOR THE RCS OF A BURIED TARGET}

In this study, it is assumed that a rectangular target with dimensions $\Delta x \times \Delta y \times h$, permeability $\mu_{0}$, and complex permittivity $\epsilon_{2}$ is buried at depth $d$ inside a soil background medium with permeability $\mu_{0}$ and complex permittivity $\epsilon_{1}$. The geometry for a perpendicular polarized plane wave incident at angle $\theta_{i}$ with respect to the $z$ axis is shown in Fig. 1 . The goal of this study is to determine the RCS of the target by using the PO approximation on the top surface of the target. The target RCS will also be computed when the target is in free space and the ratio of buried/nonburied RCS considered to clarify soil medium effects. In both cases, total electric and magnetic fields at the top surface of the target must be determined to compute the equivalent electric and magnetic current densities of the target, and

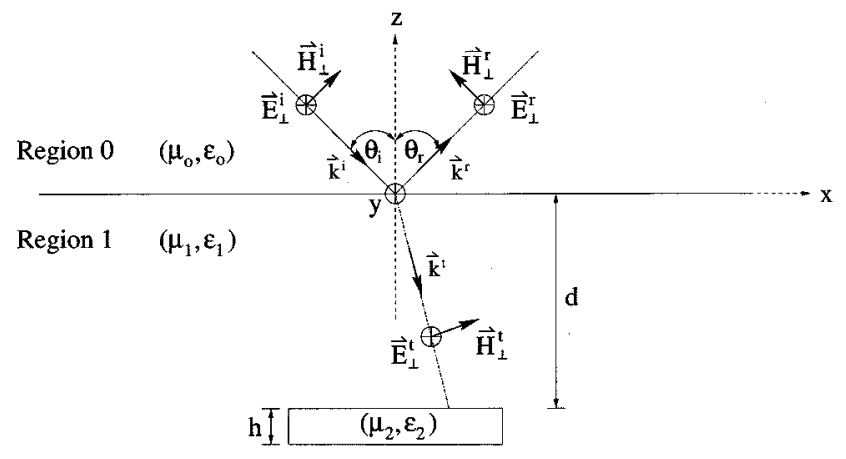

Fig. 1. Geometry for oblique incidence reflection: Perpendicular polarization.

these equivalent current densities are used to calculate the electric and magnetic vector potentials. The scattered electric field in the far zone can then be computed from these vector potentials. Multiple reflections between the ground and target surface are neglected in this analysis.

According to Fig. 1, a perpendicular polarized incident electric and magnetic field in region 0 can be written as

$$
\begin{aligned}
\vec{E}_{\perp}^{i} & =\hat{y} E_{0} e^{-j\left(k_{x}^{i} x-k_{z}^{i} z\right)} \\
\vec{H}_{\perp}^{i} & =\left(\frac{\hat{x} k_{z}^{i}+\hat{z} k_{x}^{i}}{\omega \mu_{0}}\right) E_{0} e^{-j\left(k_{x}^{i} x-k_{z}^{i} z\right)}
\end{aligned}
$$

where $E_{0}$ is the amplitude of the incident electric field and an $e^{j \omega t}$ time convention is used. The total electric and magnetic fields in the soil medium (region 1) are modeled as a combination of the transmitted fields and fields reflected from the target. In the PO approximation, reflections from the top surface of the target are modeled as reflections from an infinite layer, so that total fields in region 1 are written as

$$
\begin{aligned}
\vec{E}_{\perp}= & \hat{y} T_{\perp} E_{0} e^{-j\left(k_{x}^{i} x-k_{z}^{t} z\right)}+\hat{y} \tilde{\Gamma}_{\perp}^{\prime} T_{\perp} E_{0} e^{-j\left(k_{x}^{i} x+k_{z}^{t} z\right)} \\
\vec{H}_{\perp}= & \left(\frac{\hat{x} k_{z}^{t}+\hat{z} k_{x}^{i}}{\omega \mu_{0}}\right) T_{\perp} E_{0} e^{-j\left(k_{x}^{i} x-k_{z}^{t} z\right)} \\
& +\left(\frac{-\hat{x} k_{z}^{t}+\hat{z} k_{x}^{i}}{\omega \mu_{0}}\right) \tilde{\Gamma}_{\perp}^{\prime} T_{\perp} E_{0} e^{-j\left(k_{x}^{i} x+k_{z}^{t} z\right)}
\end{aligned}
$$

where $T_{\perp}$ is the transmission coefficient from region 0 to region 1

$$
T_{\perp}=\frac{2 k_{z}^{i}}{k_{z}^{i}+k_{z}^{t}} .
$$

$\tilde{\Gamma}_{\perp}^{\prime}$ is the reflection coefficient at the top surface of the target which can be derived by using the usual multilayer boundary condition procedure for the top surface of a dielectric slab with thickness $h$ located at distance $d$ below the ground surface

$$
\begin{aligned}
& \tilde{\Gamma}_{\perp}^{\prime}=\tilde{\Gamma}_{\perp} e^{-j 2 k_{z}^{t} d} \\
& \tilde{\Gamma}_{\perp}=\frac{\left(\tilde{k}_{z}^{t^{2}}-k_{z}^{t}\right)\left(1-e^{-j 2 \tilde{k}_{z}^{t} h}\right)}{\left(\tilde{k}_{z}^{t}-k_{z}^{t}\right)^{2} e^{-j 2 \tilde{k}_{z}^{t} h}-\left(\tilde{k}_{z}^{t}+k_{z}^{t}\right)^{2}} .
\end{aligned}
$$


Note that the propagation constants in each region are defined as

$$
\begin{aligned}
k_{x}^{i} & =\omega \sqrt{\mu_{0} \epsilon_{0}} \sin \theta_{i} \\
k_{z}^{i} & =\omega \sqrt{\mu_{0} \epsilon_{0}} \cos \theta_{i} \\
k_{z}^{t} & =\sqrt{\omega^{2} \mu_{0} \epsilon_{1}-\omega^{2} \mu_{0} \epsilon_{0} \sin ^{2} \theta_{i}} \\
\tilde{k}_{z}^{t} & =\sqrt{\omega^{2} \mu_{0} \epsilon_{2}-\omega^{2} \mu_{0} \epsilon_{0} \sin ^{2} \theta_{i}} .
\end{aligned}
$$

Under the PO approximation, the equivalent electric and magnetic current densities at the top surface of the target are

$$
\begin{aligned}
\vec{J}_{p} & =\hat{y} \frac{k_{z}^{t} T_{\perp} E_{0}}{\omega \mu_{0}}\left[1-\tilde{\Gamma}_{\perp}\right] e^{-j\left(k_{x}^{i} x+k_{z}^{t} d\right)} \\
\vec{M}_{p} & =\hat{x} T_{\perp} E_{0}\left[1+\tilde{\Gamma}_{\perp}\right] e^{-j\left(k_{x}^{i} x+k_{z}^{t} d\right)}
\end{aligned}
$$

and backscattered electric and magnetic vector potentials in the far field are approximately

$$
\begin{aligned}
& \vec{A} \simeq \frac{\mu_{0} e^{-j k_{0} r} e^{-j k_{z}^{t} d} \tilde{T}_{\perp}}{4 \pi r} \int_{-\frac{\Delta x}{2}}^{\frac{\Delta x}{2}} \int_{-\frac{\Delta y}{2}}^{\frac{\Delta y}{2}} \vec{J}_{p} e^{-j k_{x}^{i} x^{\prime}} d x^{\prime} d y^{\prime} \\
& \vec{F} \simeq \frac{\epsilon_{1} e^{-j k_{0} r} e^{-j k_{z}^{t} d} \tilde{T}_{\perp}}{4 \pi r} \int_{-\frac{\Delta x}{2}}^{\frac{\Delta x}{2}} \int_{-\frac{\Delta y}{2}}^{\frac{\Delta y}{2}} \vec{M}_{p} e^{-j k_{x}^{i} x^{\prime}} d x^{\prime} d y^{\prime}
\end{aligned}
$$

Here $r$ is the distance between the origin and the observation point, and $k_{0}$ is the propagation constant in free space equal to $\omega \sqrt{\mu_{0} \epsilon_{0}}$. Also $\tilde{T}_{\perp}=2 k_{z}^{t} /\left(k_{z}^{t}+k_{z}^{i}\right)$ is the transmission coefficient from region 1 to 0 . Equations (14) and (15) are constructed from the basic idea that the target is replaced by the equivalent electric and magnetic current sources under the PO approximation. The electric and magnetic fields radiated by these sources in the soil medium then propagate until meeting the interface between the soil medium and free space, at which point the transmission coefficient from region 1 to 0 is included. Substituting $\vec{J}_{p}$ and $\vec{M}_{p}$ from (12) and (13) into (14) and (15) and computing the integral, the vector potentials are shown in (16) and (17) at the bottom of the page. Scattered electric fields corresponding to these electric and magnetic vector potentials are shown in (18) and (19) at the bottom of the page, where $\eta_{1}=\sqrt{\mu_{0} / \epsilon_{1}}$ is the intrinsic impedance of the soil medium. Therefore, the total scattered electric field can be written as

$$
\begin{aligned}
\vec{E}_{\perp}^{s} & =\vec{E}_{A}^{s}+\vec{E}_{F}^{s} \\
& =\hat{y} \frac{k_{z}^{t} T_{\perp} E_{0} \tilde{T}_{\perp} e^{-j k_{0} r} e^{-j 2 k_{z}^{t} d} \Delta x \Delta y \sin \left(k_{x}^{i} \Delta x\right) 2 \tilde{\Gamma}_{\perp}}{4 \pi r k_{x}^{i} \Delta x} .
\end{aligned}
$$

For normal incidence, (21) becomes

$$
\vec{E}_{\perp}^{s}=\hat{y} \frac{k_{1} T_{\perp} E_{0} \tilde{T}_{\perp} e^{-j k_{0} r} e^{-j 2 k_{1} d} \Delta x \Delta y 2 \tilde{\Gamma}_{\perp}}{4 \pi r} .
$$

The backscattering RCS is

$$
\sigma=\lim _{r \rightarrow \infty}\left[\frac{4 \pi r^{2}\left|\vec{E}^{s}\right|^{2}}{\left|\vec{E}^{i}\right|^{2}}\right]
$$

Substituting $\vec{E}_{\perp}^{i}$ and $\vec{E}_{\perp}^{s}$ from (1) and (22) into (23), the normal incidence RCS becomes

$$
\sigma_{\perp}=\left(\left|k_{1} T_{\perp} \tilde{T}_{\perp} \tilde{\Gamma}_{\perp}\right|^{2} e^{-4 \Im\left[k_{1} d\right]}\right)\left(\frac{\Delta x^{2} \Delta y^{2}}{\pi}\right)\left|Q^{2}\right|
$$

where $k_{1}$ is the propagation constant in the soil medium equal to $\omega \sqrt{\mu_{0} \epsilon_{1}}$ and $\Im$ indicates the imaginary part. An additional factor $Q^{2}$ has been introduced in (24) to account for modification of the power density per unit angle of the scattered spherical wave due to refraction through the interface. Consideration of the plane wave representation of a spherical wave shows that the factor $Q=k_{z}^{i} / k_{z}^{t}$. Note that the effect of spherical wave propagation between the two boundaries will cancel out if the incident wave from the source in the air region is a spherical wave and not a plane wave. $Q$ can thus be written as

$$
\begin{aligned}
Q & =\frac{k_{z}^{i}}{k_{z}^{t}} \quad \text { for plane wave incidence } \\
& =1 \text { for spherical wave incidence. }
\end{aligned}
$$

For a target in free space, $T_{\perp}=\tilde{T}_{\perp}=1$, and the reflection coefficient at the top surface of the target $\left(\tilde{\Gamma}_{\perp}\right)$ from (7) is written as $\tilde{\Gamma}_{\perp}^{0}$. The normal incidence backscattering RCS of the target in free space can then be written as

$$
\sigma_{\perp}^{0}=\left|k_{0} \tilde{\Gamma}_{\perp}^{0}\right|^{2}\left(\frac{\Delta x^{2} \Delta y^{2}}{\pi}\right) .
$$

$$
\begin{aligned}
& \vec{A}=\hat{y} \frac{k_{z}^{t} T_{\perp} E_{0}\left[1-\tilde{\Gamma}_{\perp}\right] \tilde{T}_{\perp} e^{-j k_{0} r} e^{-j 2 k_{z}^{t} d} \Delta x \Delta y \sin \left(k_{x}^{i} \Delta x\right)}{4 \pi r \omega k_{x}^{i} \Delta x}=\hat{y} A \\
& \vec{F}=\hat{x} \frac{\epsilon_{1} T_{\perp} E_{0}\left[1+\tilde{\Gamma}_{\perp}\right] \tilde{T}_{\perp} e^{-j k_{0} r} e^{-j 2 k_{z}^{t} d} \Delta x \Delta y \sin \left(k_{x}^{i} \Delta x\right)}{4 \pi r k_{x}^{i} \Delta x}=\hat{x} F .
\end{aligned}
$$

$$
\begin{aligned}
& \vec{E}_{A}^{s} \simeq-\hat{y} j \omega A \simeq-\hat{y} \frac{j \omega k_{z}^{t} T_{\perp} E_{0}\left[1-\tilde{\Gamma}_{\perp}\right] \tilde{T}_{\perp} e^{-j k_{0} r} e^{-j 2 k_{z}^{t} d} \Delta x \Delta y \sin \left(k_{x}^{i} \Delta x\right)}{4 \pi r \omega k_{x}^{i} \Delta x} \\
& \vec{E}_{F}^{s} \simeq \hat{y} \eta_{1} j \omega F \simeq \hat{y} \frac{j \omega \eta_{1} k_{z}^{t} \epsilon_{1} T_{\perp} E_{0}\left[1+\tilde{\Gamma}_{\perp}\right] \tilde{T}_{\perp} e^{-j k_{0} r} e^{-j 2 k_{z}^{t} d} \Delta x \Delta y \sin \left(k_{x}^{i} \Delta x\right)}{4 \pi r \omega \sqrt{\mu_{0} \epsilon_{2}} k_{x}^{i} \Delta x}
\end{aligned}
$$



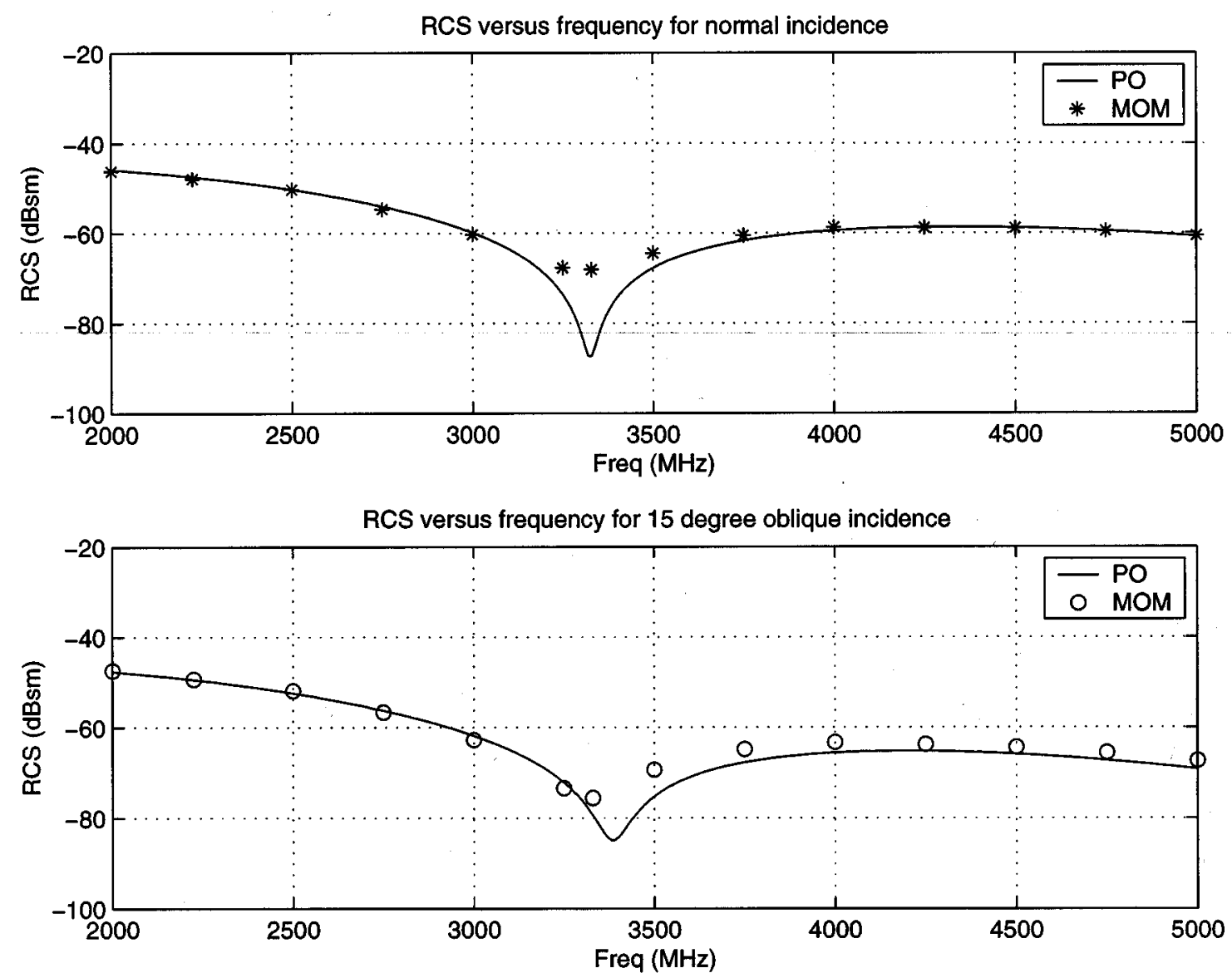

Fig. 2. Comparison of analytically calculated RCS from PO and numerically calculated RCS from MoM versus frequency for normal and 15 degree oblique incidence.

Finally, the ratio between the normal incidence target RCS in the soil medium and in free space can be calculated by dividing (24) by (27) to obtain

$$
\frac{\sigma_{\perp}}{\sigma_{\perp}^{0}}=\left|\frac{k_{1} \tilde{T}_{\perp} T_{\perp} \tilde{\Gamma}_{\perp}}{k_{0} \tilde{\Gamma}_{\perp}^{0}}\right|^{2} e^{-4 \Im\left[k_{1} d\right]}\left|Q^{2}\right| .
$$

Results for parallel polarization can be derived in a similar manner and are not detailed here; the emphasis of the current study on near normal incidence should result in only slight differences between polarizations.

Obviously from (28), the ratio of normal incidence backscattered RCS of a target in the soil medium with respect to that for a a target in free space is dependent on reflection and transmission coefficients and on attenuation in the background medium. The reflection coefficient term $\left|\tilde{\Gamma}_{\perp}\right|^{2}$ clearly will increase as the dielectric contrast between the target and soil medium becomes larger, although the transmission coefficients $\left|\tilde{T}_{\perp} T_{\perp}\right|^{2}$ will tend to decrease as background medium contrast with free space increases.

To validate this simple PO model, the backscattered RCS of a target for 0 degree and 15 degree plane wave incidence on a soil medium was calculated and compared with numerical results from a MoM code [12]. In this validation a nylon target $\left(\epsilon_{r} \approx 3.15-j 0.03\right.$ from $\mathrm{S}$-band waveguide measurements described in [2]) with cross section $7.62 \mathrm{~cm}$ by 7.62 $\mathrm{cm}$ and thickness of $2.54 \mathrm{~cm}$ was assumed to be buried at
$7.62 \mathrm{~cm}$ below the ground surface. Soil with dielectric constant $5-j 1.25$ (from S-band waveguide measurements of homogeneous sand with $7.5 \%$ water content [2]) was used as the background medium. The frequency range was chosen to be $2-5 \mathrm{GHz}$. Fig. 2 shows the comparison of the analytically calculated RCS from the PO model and the numerically calculated RCS from the MoM for both normal and 15 degree oblique incidence (using the fields of (21) in the PO model at oblique incidence) in perpendicular polarization. The analytical data were computed in $0.01 \mathrm{GHz}$ steps whereas the numerical data used $0.25 \mathrm{GHz}$ steps, due to the much larger computational requirements of the MOM code (for example 1060 unknowns used at $4 \mathrm{GHz}$, requiring $2.5 \mathrm{~h} \mathrm{CPU} \mathrm{time}$ on a $200-\mathrm{MHz}$ Pentium Pro processor). From the plot a null is observed in $\mathrm{PO}$ predictions at frequency $3330 \mathrm{MHz}$ due to a resonance in the dielectric target reflection coefficient. Clearly the analytical and numerical data match reasonably well. The average level of error excluding the null frequency is about 0.77 and $1.43 \mathrm{~dB}$ for normal and 15 degree oblique incidence, respectively. Fig. 3 demonstrates another comparison of analytically calculated RCS from the PO model and numerically calculated RCS from the MoM versus incidence angle at $4 \mathrm{GHz}$ for perpendicular and parallel polarizations. Again, both analytical and numerical data show reasonable agreement, although the differences increase at more oblique angles as expected for a PO model. These results demonstrate 

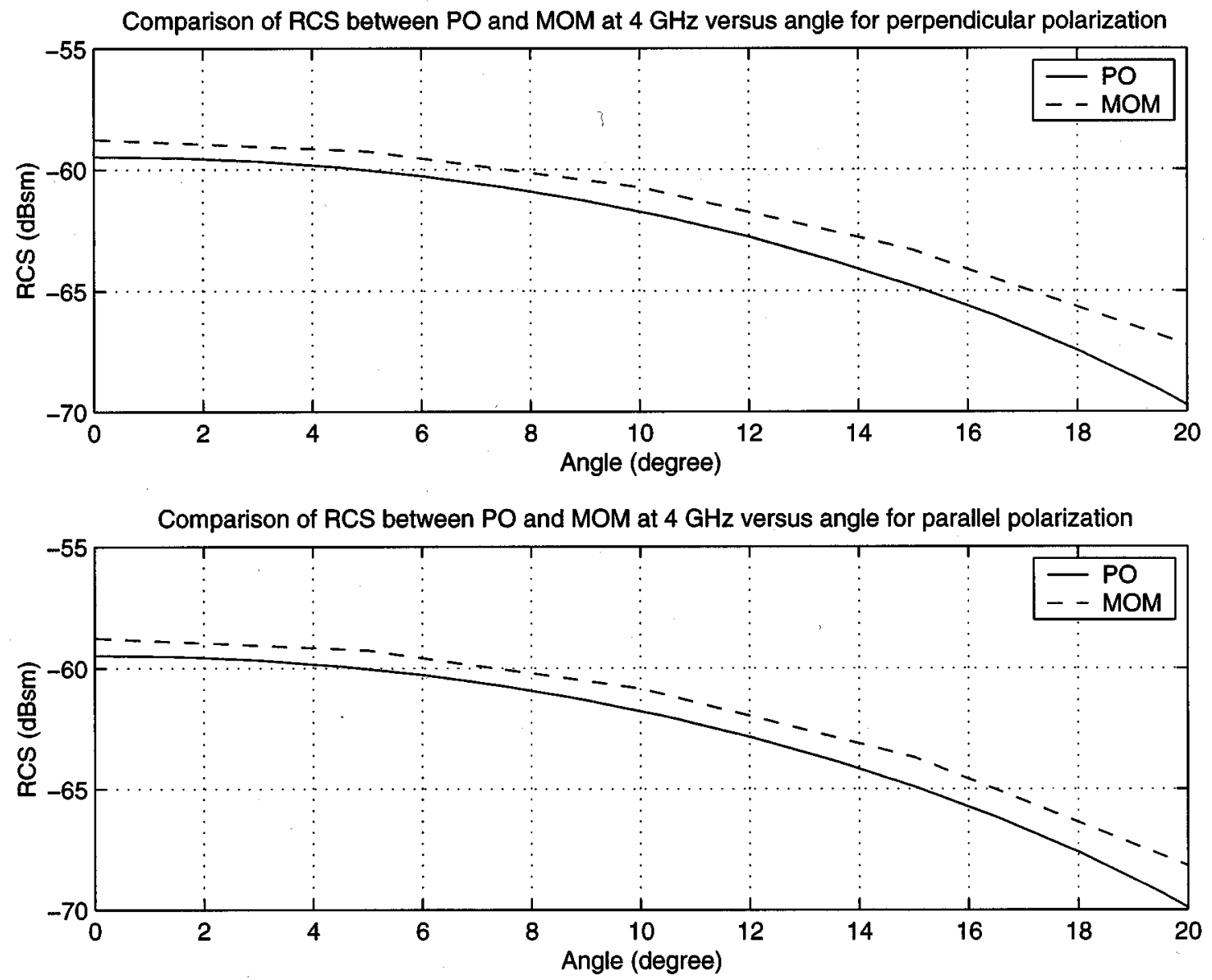

Fig. 3. Comparison of analytically calculated RCS from PO and numerically calculated RCS from MoM versus angle at $4 \mathrm{GHz}$ for perpendicular and parallel polarizations.

that a simple PO model can be valid for RCS studies of a buried target at near normal incidence angles.

A comparison between the PO model and a GPR measurement is next considered. The experimental result was obtained using a dielectric rod antenna GPR system [13], depicted in Fig. 4. A network analyzer was set to transmit continuous wave (CW) electromagnetic signals with IF bandwidth $1000 \mathrm{~Hz}$ and power $10 \mathrm{dBm}$ in $0.1 \mathrm{GHz}$ steps from 2 to $6 \mathrm{GHz}$. The dielectric rod antenna was located at 1 in above the ground surface in order to obtain a very small illuminated area, and attached to a horizontal movement controller so that measurements could be taken as a function of position. The system has a basic calibration procedure involving two small cylindrical wire scatterers, but the near field nature of this system makes a complete calibration difficult so only qualitative image responses are compared. The target considered in this measurement was a $7.62-\mathrm{cm} \mathrm{di-}$ ameter nylon disk of $2.54 \mathrm{~cm}$ thickness buried at $6.35 \mathrm{~cm}$ depth. The measured result is presented in Fig. 5(a) while the analytical result is presented in Fig. 5(b). The theoretical result was generated using (21) (with $Q=1$ for spherical wave incidence) to calculate the scattered electric field assuming that the dielectric constants of soil and nylon are the same as in the previous case except that the target was buried at 2.5 -in depth. In this study, clutter returns from the ground surface which occur in measured early time images (which in practice require more advanced suppression methods [14]) are not included in the analytical model. To model the rod antenna movement, additional amplitude and phase factors must be included in (21). According to Fig. 6 which illustrates the geometry for the rod antenna movement, the factor $e^{-j 2 d k_{z}^{t}}$ in (21) should be replaced by $\left[e^{-\left|2 \Im\left[k_{z}^{t}\right] d\right|} e^{-j 2\left(k_{x}^{i} a+\Re\left[k_{z}^{t}\right] d\right)} e^{-j 2 k_{0} s_{1}}\right] /\left[s_{1}+s_{2}\right]$ to take into account attenuation in the soil medium, spherical wave propagation, and phase shifts in both media; here $\Re$ indicates real part. Here $h$ is the vertical distance from the tip of the rod antenna to the ground surface, $x$ is the horizontal distance from the tip of the rod antenna to the center of the target and $d$ is the depth of the target, all of which are known. The parameters $a, s_{1}$, and $s_{2}$ are determined by

$$
\begin{aligned}
s_{1} & =\sqrt{h^{2}+b^{2}} \\
s_{2} & =\sqrt{d^{2}+a^{2}} \\
b & =h \tan \theta_{i} \\
a & =x-b \\
x & =h \tan \theta_{i}+d \tan \theta_{t}=h \tan \theta_{i}+\frac{d \sin \theta_{i}}{\Re\left[\sqrt{\frac{\epsilon_{1}}{\epsilon_{0}}-\sin ^{2} \theta_{i}}\right]} .
\end{aligned}
$$

The parameter $\theta_{t}$ in (33) is not the transmitted angle, which is a complex number, but the angle at which the constant phase 


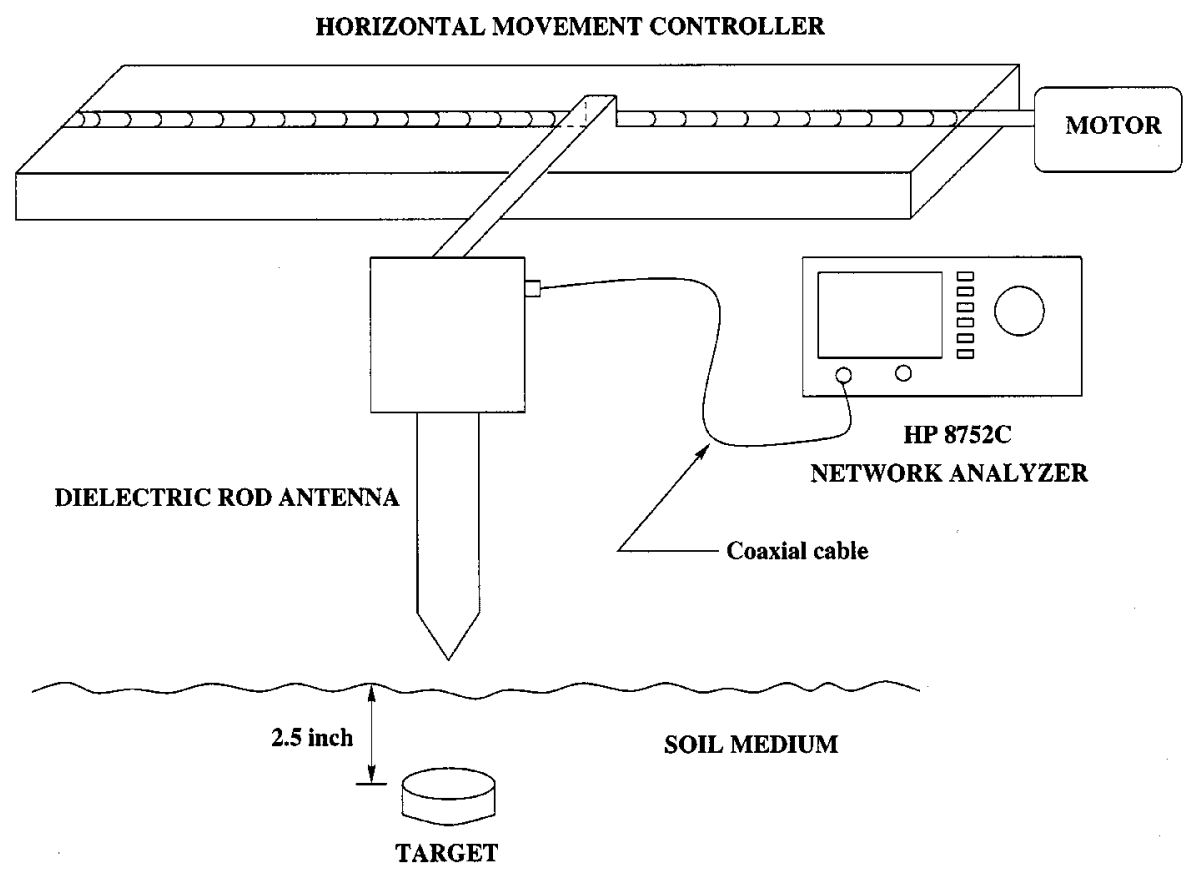

Fig. 4. Experimental configuration for dielectric rod antenna GPR system.

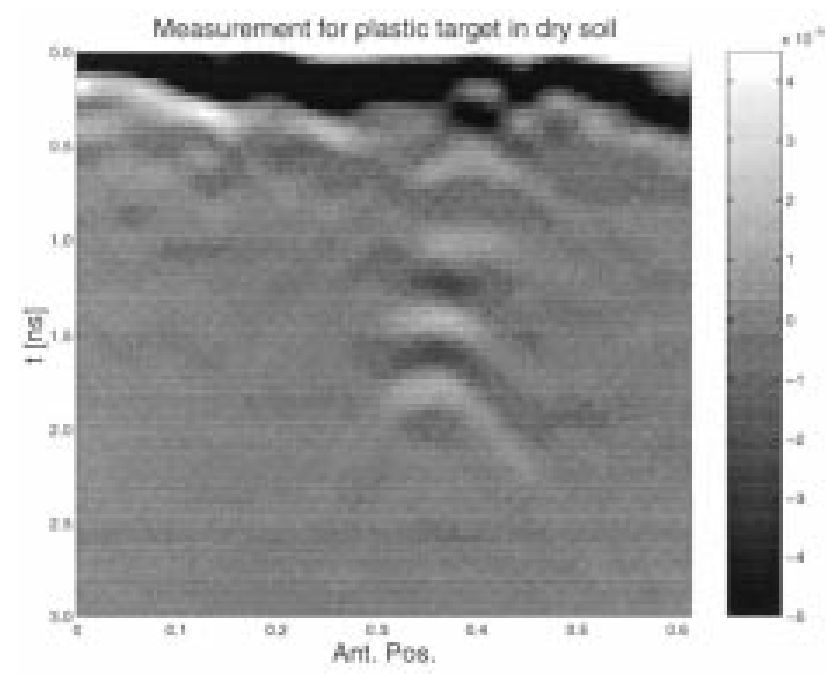

(a)

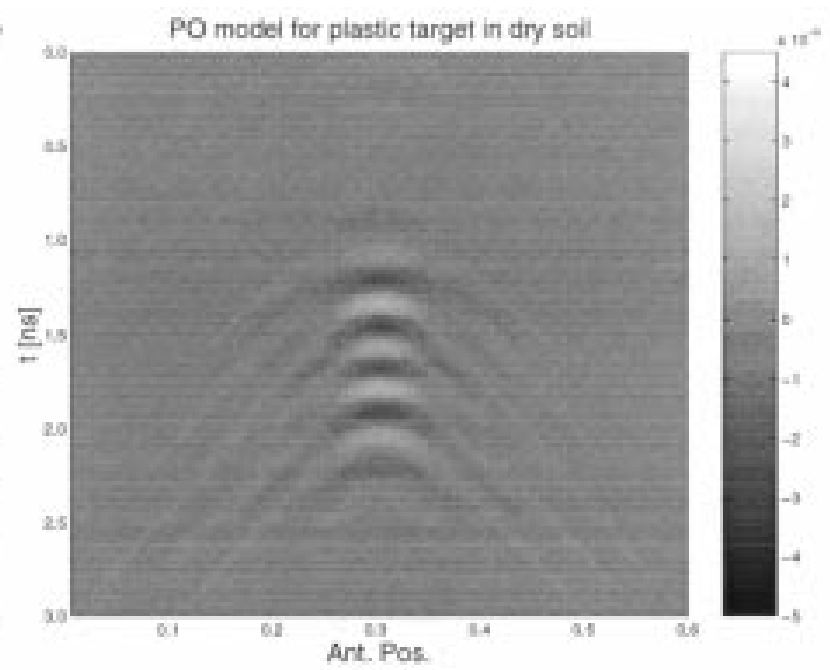

(b)

Fig. 5. Comparison of time domain image scattered fields from PO model and GPR measurement: plastic target $\left(\epsilon_{r}=3.15-j 0.03\right)$ buried at 2.5 -in depth in soil $\left(\epsilon_{r}=5-j 1.25\right)$; field units are defined such that their magnitude squared in the frequency domain is the radar cross section in square meters. (a) Measurement. (b) PO model.

planes of the transmitted plane wave are inclined at with respect to the $z$ axis. The parameter $\theta_{i}$ is found by numerically solving the nonlinear equation (33). Both images in Fig. 5 are produced by plotting time domain scattered fields as a function of antenna horizontal position, and results are calibrated so that time $0 \mathrm{nS}$ corresponds to the top surface of the ground. Note that both images are plotted with the same amplitude scale. As observed from the plot, features of both results are similar, with target responses occuring around the same time; however more oscillations are observed at later times in theoretical model results. In the theoretical model, the permittivity of the soil medium is assumed to be constant but the natural soil medium in the measurement is not homogeneous, so that it is possible to obtain for example more loss for deeper soil. However, since these two

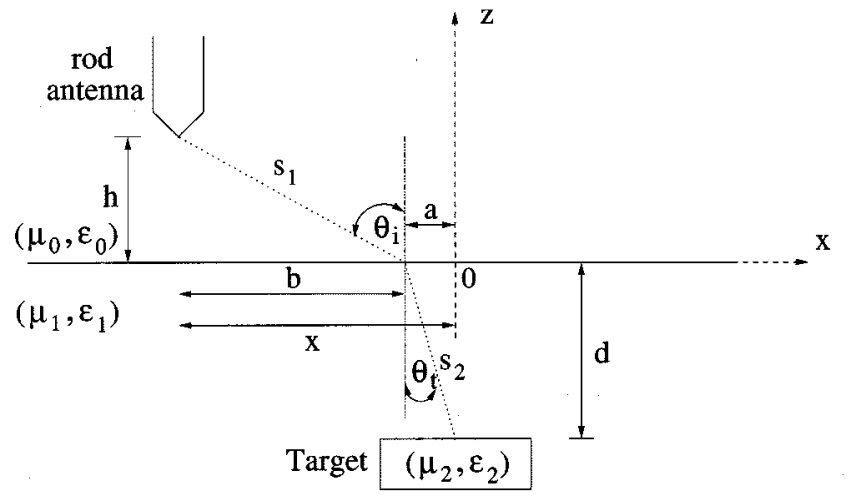

Fig. 6. Geometry for modeling dielectric rod antenna movement. 
A
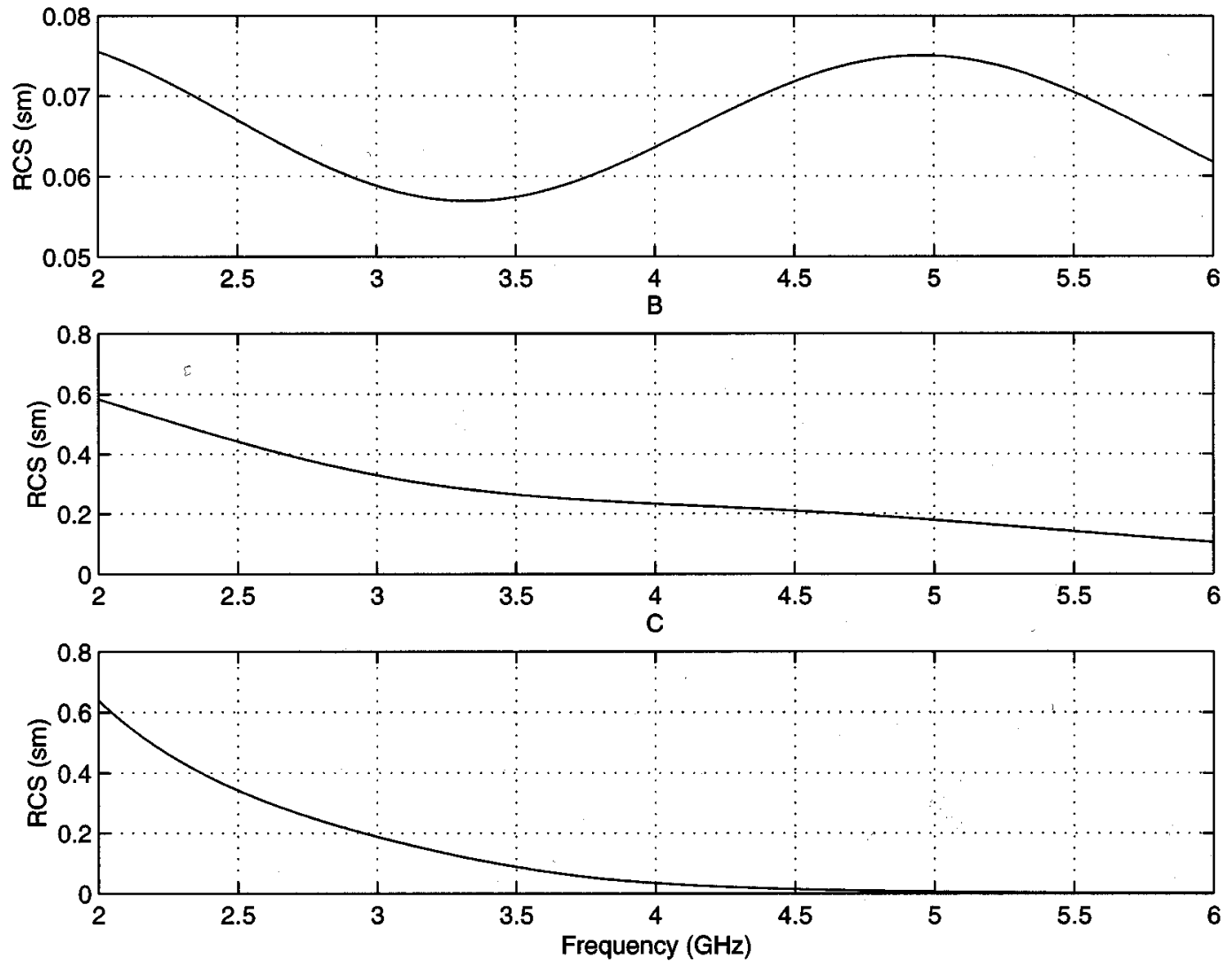

Fig. 7. Ratio of backscattering RCS of nylon target versus frequency for normal incidence in soil medium with respect to free space when soil medium is (A) dry sand (B) sand with $7.5 \%$ water content (C) sand with $30 \%$ water content.

images are in good qualitative agreement in general, this comparison also supports the validation of the PO model. The following section will concentrate on an analytical study of target detection using the PO model.

\section{ANAlytical StUdy OF TARGEt Detection}

To demonstrate that the RCS of a buried target depends on the dielectric contrast between the target and background medium, (28) was used to generate the ratio of backscattering RCS of a target in a homogeneous water content soil medium with respect to free space for normally incident spherical waves. In this study the nylon target $\left(\epsilon_{r} \approx 3.15-j 0.03\right)$ with thickness of $2.54 \mathrm{~cm}$ was assumed to be buried at $7.62 \mathrm{~cm}$ below the top ground surface. Sand with three different homogeneous water contents was used as the background medium: dry sand $\left(\epsilon_{r} \approx 2.6-j 0.005\right)$, sand with $7.5 \%$ water content $\left(\epsilon_{r} \approx 5.8-j 0.3\right)$ and sand with $30 \%$ water content $\left(\epsilon_{r} \approx 17.8-j 2\right)$; these permittivity values again were obtained from $S$-band waveguide measurements [2]. Dry sand was used in the case of no modification and expected to produce a small RCS. Sand with $7.5 \%$ water content was used in the case of "optimal" water modification and expected to produce larger RCS. Sand with $30 \%$ water content was used in the case of excessive water modification and expected to produce a small RCS again. Fig. 7 illustrates the simulated ratio of backscattering RCS with respect to free space of the nylon target versus frequency for normal incidence in the three soil media.
The frequency range was chosen to be $2-6 \mathrm{GHz}$, and analytical data were calculated in $0.01-\mathrm{GHz}$ steps. Plot (A) shows that the RCS of the plastic target in dry sand is small because of the low dielectric contrast. In plot (B), the RCS of the plastic target is larger than that in plot (A) since there is more dielectric contrast in this case; note the scale in plot (B) has been increased by a factor of ten. The RCS of plot (B) is around 4.5 times that of plot (A) at the center frequency $(4 \mathrm{GHz})$. In plot (C) the RCS of the plastic target becomes obscured at high frequencies because electromagnetic wave absorption is significant at high frequencies. Even though the dielectric contrast in this case is larger than that in plot (B), loss in the background medium prohibits the incident wave from reaching the target so that the RCS is still low. However, at low frequencies it could be possible to detect the target since the loss is small compared to the loss at high frequencies. Clearly the RCS of the target in this plot is close to that in plot (A) at the center frequency. These results again demonstrate the dependence of buried target RCS on the dielectric contrast with and attenuation in the background medium.

The concept of soil modification through the addition of water to obtain an "optimal" homogeneous soil water content is next considered in more detail. The optimal homogeneous soil water content is defined here as the value which gives the largest peak backscattered field response in the time domain. An empirical model [15] is used to estimate the relative permittivity of soils for a given homogeneous water content and frequency and requires soil physical parameters such as volumetric moisture and 


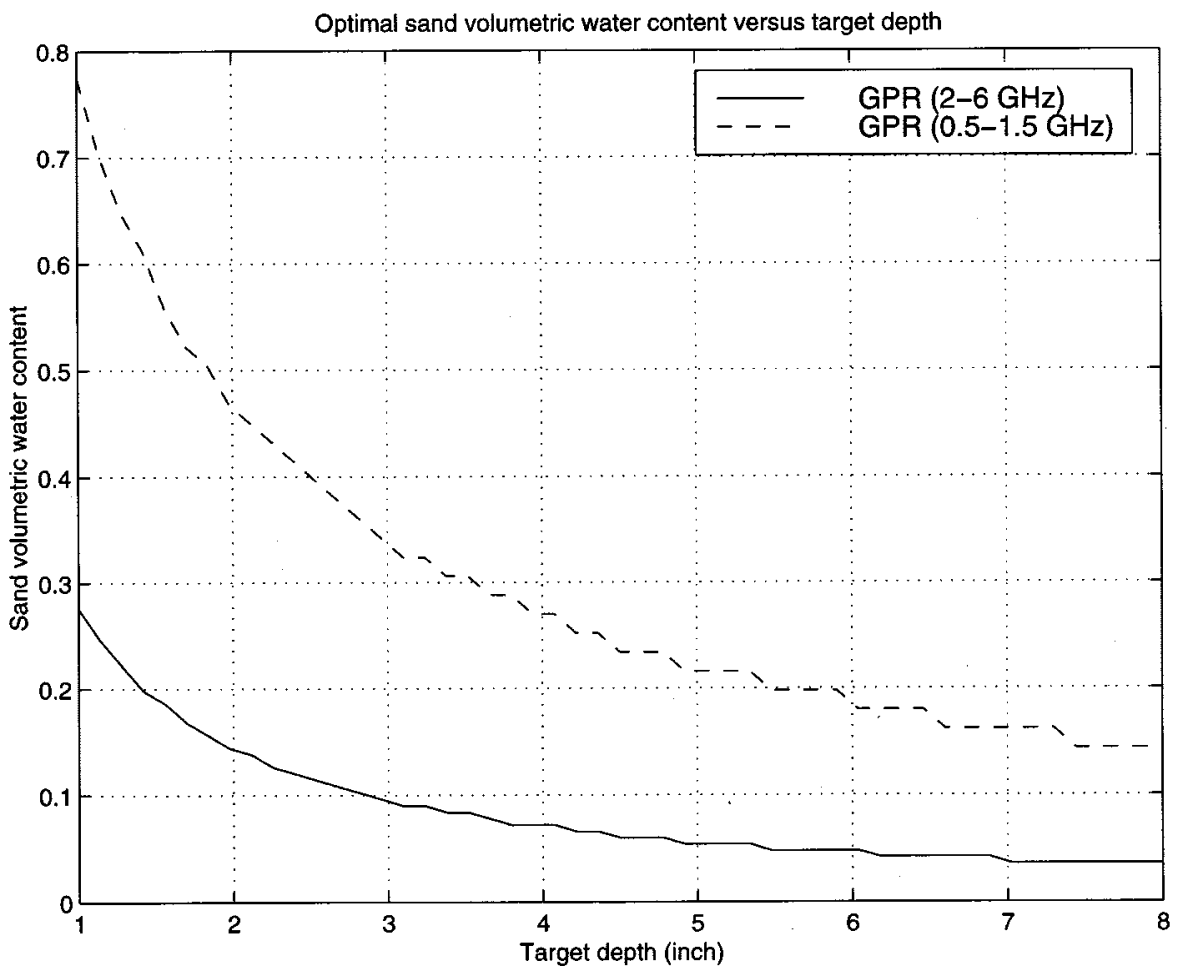

Fig. 8. Optimal sand volumetric water content obtained in analytical study versus target depth.

soil textural composition as input data. The analytical PO model is used along with the dielectric constant from this empirical model to perform a theoretical study of the maximum target response for each water content. Fig. 8 illustrates optimal homogeneous sand (bulk density $=1.55 \mathrm{~g} / \mathrm{cm}^{3}$ ) water contents obtained as a function of target depth. In this study, the target depths were varied from 1 to 8 in $(0.0254$ to $0.2032 \mathrm{~m})$, and the relative permittivity of the nylon target was assumed to be $3-j 0.08$. Two frequency ranges $(2-6 \mathrm{GHz}$ and $0.5-1.5 \mathrm{GHz}$ ) were chosen to generate this plot. As observed from the plot, optimal water contents decrease when frequency increases because loss is more significant at higher frequencies. Optimal homogeneous soil water contents also decrease when target depths increase since loss even in the lower water content soil will become significant for very deep targets, and in many cases it may be difficult to obtain the optimal water content. However, the addition of liquid nitrogen onto a soil medium can reduce loss and restore target visibility due to the much smaller loss tangents in ice than water.

Fig. 9 illustrates soil modification through the addition of water and liquid nitrogen procedure proposed to locate targets at a known depth. In plot (A) of Fig. 9, a low contrast target is buried at a known depth $d$ in dry sand. Scattered returns in plot (A) would be small due to the small dielectric contrast between the plastic target and dry sand medium. The second figure of plot (A) shows the configuration after a large amount of water is added onto the top sand surface. Scattered returns in this case would again be small due to an excessive attenuation in the very wet sand above the target. Fig. 9 plots (B) through (D) now illustrate the configuration as increasing amounts of liquid nitrogen are added, so that the frozen sand region above the target extends

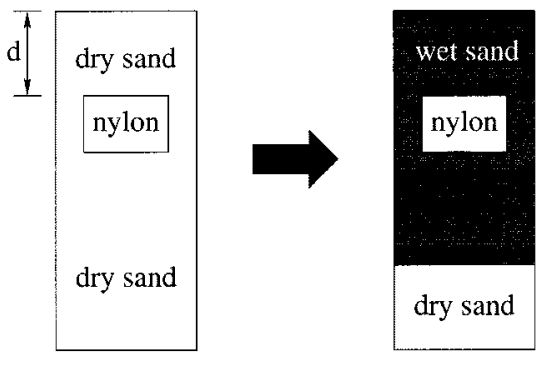

Before modification

Adding water

(a)

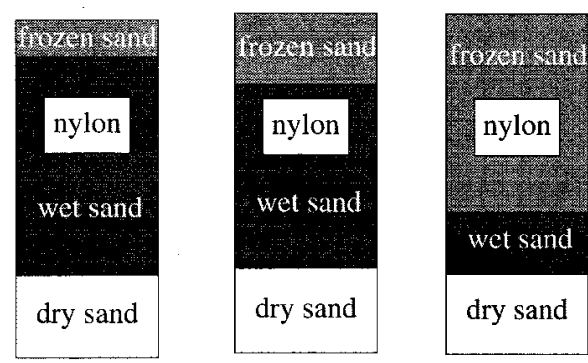

Adding insufficient Adding optimal Adding excessive liquid nitrogen liquid nitrogen liquid nitrogen

(b)

(c)

(d)

Fig. 9. Soil modification through the addition of water and liquid nitrogen.

to greater and greater depths. In plot (B) only a small quantity of nitrogen has been added so that loss in the wet sand region above the target still causes excessive attenuation and the target remains obscured. In plot (C), an "optimal" amount of liquid nitrogen has been added so that loss above the target is mini- 


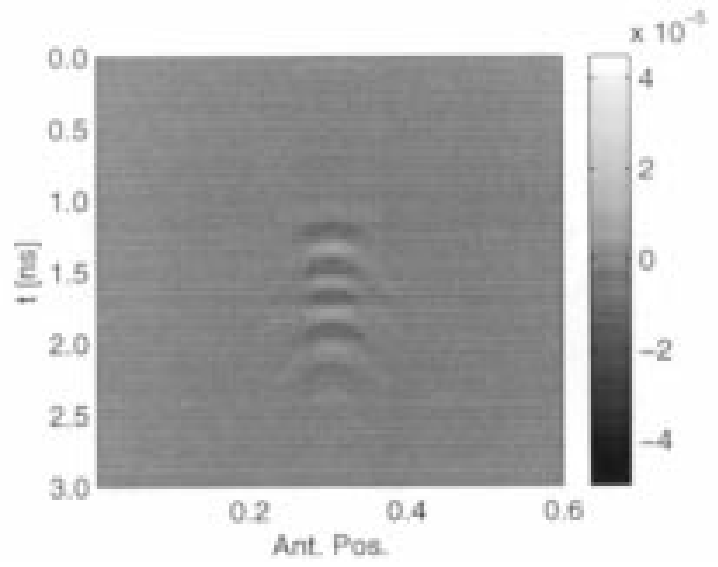

(a)

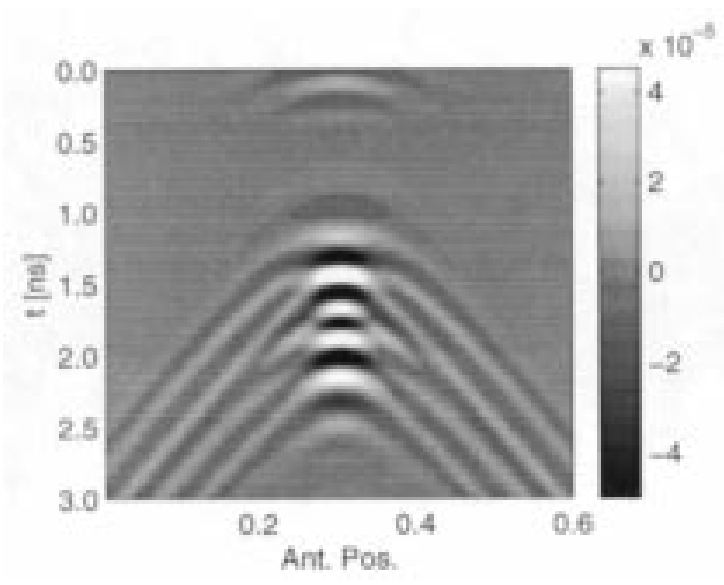

(c)

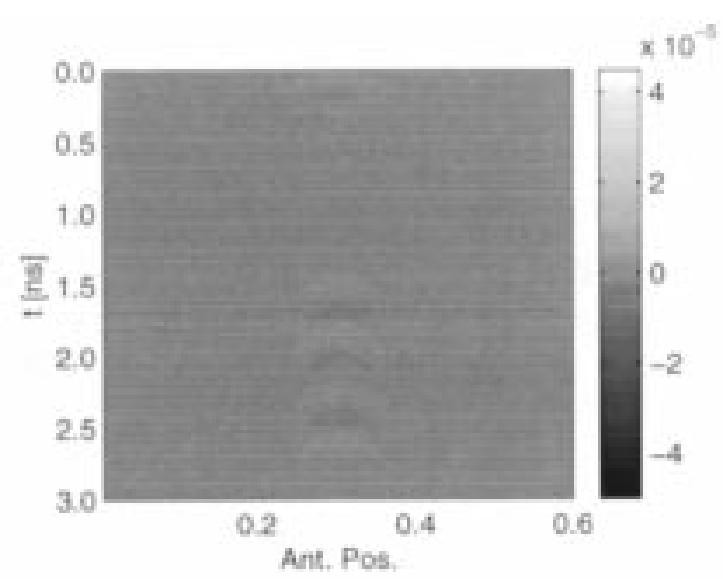

(b)

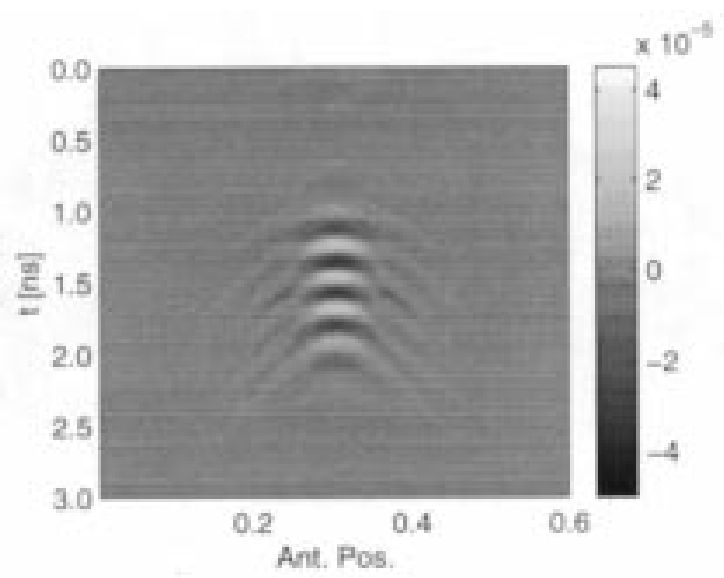

(d)

Fig. 10. Image results of time domain simulated scattered fields from PO model for the configurations of Fig. 8 with $6.35 \mathrm{~cm}$ depth nylon target. (a) Target in dry soil. (b) After the addition of water and a small amount of liquid nitrogen. (c) After the addition of an "optimal" amount of liquid nitrogen. (d) After the addition of an excessive amount of liquid nitrogen; field units are defined as in Fig. 5.

mized, but a large dielectric contrast in the region surrounding the target remains to provide scattering. In plot (D), an excessive amount of nitrogen has been added and again the target is obscured due to the low contrast between the nylon and frozen sand media. If the target depth is assumed known, the optimal amount of nitrogen to be added can be determined and cataloged for future measurements of similar depth targets. However, it is also clear from Fig. 9 that the procedure should be applicable to unknown depth targets as well, since nonoptimal amounts of liquid nitrogen do not produce large target returns. Thus, a procedure in which measurements are taken as gradually increasing amounts of liquid nitrogen are added should be sufficient to improve visibility of unknown depth targets as well.

Image results of time domain simulated scattered fields from the PO model based on the configurations of Fig. 9, plots (A)-(D), are shown in Fig. 10, plots (A)-(D) for a target at depth $6.35 \mathrm{~cm}$. The model was extended to include multi-layer effects by generalizing the procedure of Section II, and reflections from boundaries between frozen and wet soils are removed to highlight target scattering effects. In this simulation, the dielectric constant of "dry" soil is approximated as $5-j 1.25$, the dielectric constant of frozen soil is approximated as $4-j 0.5$, and the dielectric constant of wet soil is approximated as $17.8-j 10$. The nylon target $\left(\epsilon_{r} \approx 3.15-j 0.03\right)$ was assumed to be buried at $6.35 \mathrm{~cm}$ below soil surface. The thickness of frozen soil is assumed to be $2.54 \mathrm{~cm}$ for configuration (B), $5.08 \mathrm{~cm}$ for configuration (C), and $6.35 \mathrm{~cm}$ for configuration (D). Fig. 10, plot (A) confirms the small scattered returns obtained from the low contrast target in dry sand. Fig. 10 plot (B) illustrates the return for the configuration of Fig. 9, plot (B); here the small amount of nitrogen added is insufficient to reduce excessive background loss and the target remains obscured. Fig. 10 plot (C) shows the greatly enhanced target response obtained in the "optimal" configuration, in which 1.27 $\mathrm{cm}$ of wet sand remain above the target. Finally Fig. 10, plot (D) shows smaller responses as the entire background medium becomes frozen.

To confirm the soil modification procedure through the addition of water and liquid nitrogen suggested by the analytical models, measurements with the dielectric rod antenna GPR system were performed using natural soil in the backyard of the ElectroScience Laboratory. The experimental configuration was the same as described previously, and both metal and nylon disks with the same dimensions as discussed in Section II buried at $6.35 \mathrm{~cm}$ depth were considered in these measurements. In this test, substantial quantities of water and liquid nitrogen were applied to ensure that soil properties were modified. Measurements of the targets were first made with the soil in its ini- 


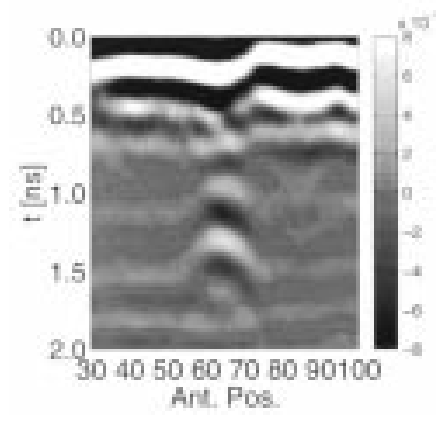

(a)

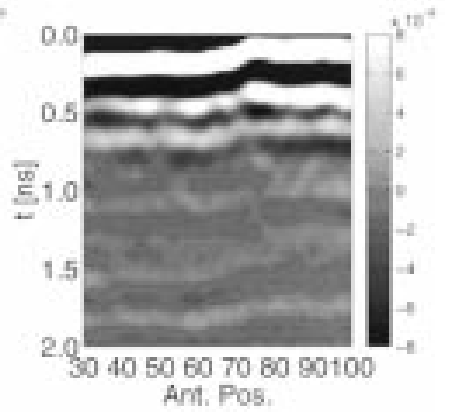

(b)

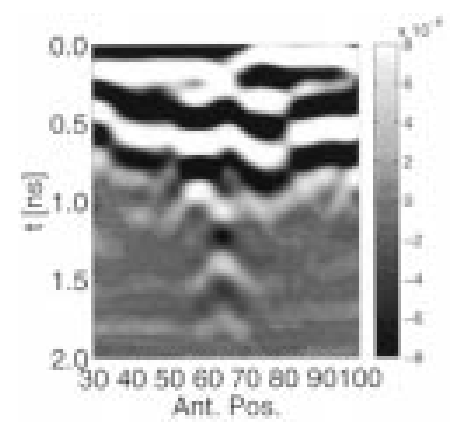

(c)

Fig. 11. Image results of time domain measured fields for dielectric rod antenna with $6.35 \mathrm{~cm}$ depth metal target. (a) Target in dry soil. (b) After the addition of 2 gallons of water. (c) After the addition of 15 liters of liquid nitrogen; field units are defined as in Fig. 5.

tial state, and then two gallons of water were poured over an area of approximately $0.5-\mathrm{m}$ square which contained the target. Measurements in this "excessively wet" configuration were then taken, and finally approximately 15 of liquid nitrogen were applied over the same area and the final measurements made. Tests of the nitrogen penetration depth were made by digging up the soil after the measurements were completed, and it was estimated that penetration to approximately $8 \mathrm{~cm}$ was obtained with this quantity of nitrogen. Although the chemical amounts used in this demonstration were substantial, it is expected that further studies can develop more efficient application procedures that will reduce the required quantities.

Measured results for the metallic and nylon targets are presented in Figs. 11 and 12, respectively as images. These images are produced by plotting time domain measured fields as a function of antenna horizontal position; the target location was known to be between antenna positions 60 and 70, and results are calibrated so that time $0 \mathrm{nS}$ corresponds to the top surface of the ground. Returns observed from 0 to $0.6 \mathrm{~ns}$ correspond to clutter and sidelobe returns from the strong surface reflections in the measurement; clutter reduction signal processing methods can be applied to reduce these contributions [14]. Figs. 11 and 12 both contain three plots: plot (a) is the image before any water or nitrogen has been added, plot (b) is the image after the addition of water, and plot (c) is image after the addition of water and liquid nitrogen. Fig. 11 plots (b) and (c) clearly demonstrate the improved visibility of a metallic target in excessively wet soil obtained after the addition of liquid nitrogen. Even though the target is visible also in the un-modified soil, liquid nitrogen addition would be useful in situations where the un-modified soil had properties closer to the wet soil considered here. Results in (a)

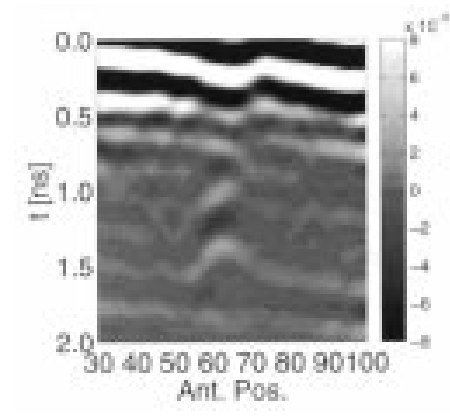

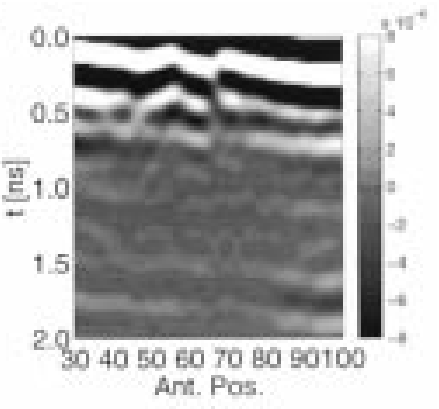

(b)

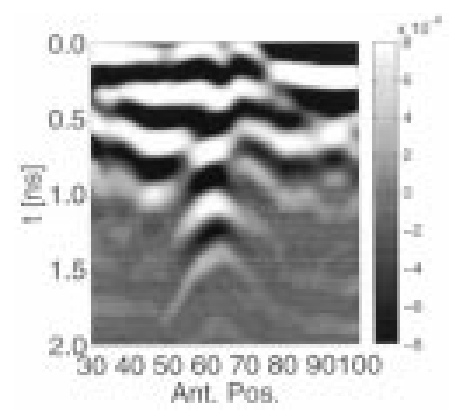

(c)

Fig. 12. Image results of time domain measured fields for dielectric rod antenna with $6.35 \mathrm{~cm}$ depth nylon target. (a) Target in dry soil. (b) After the addition of 2 gallons of water. (c) After the addition of 15 liters of liquid nitrogen; field units are defined as in Fig. 5.

Fig. 12 are also interesting: a much smaller response than the metallic target is obtained in plot (a) with the nylon target, and again the response is completely obscured in excessively wet soil. The enhanced response in part (c) again shows the effectiveness of liquid nitrogen addition. The relative contributions of reduced loss in the background and increased dielectric contrast are difficult to assess from this single measurement, but these initial qualitative results demonstrate that the soil modification techniques suggested by the analytical model should have applicability to general microwave GPR systems for detecting anti-personnel mines.

\section{SUMMARY AND CONCLUSION}

The detection of nonmetallic landmines with ground penetrating radar through soil modification has been presented. The physical optics approximation for the RCS of a buried target has been derived, and results demonstrate that the RCS of a buried target is proportional to reflection and transmission coefficients and to attenuation in the background medium. The comparison between analytical results from this simple PO model and numerical results from the method of moments validates the model for near normal incidence studies. Moreover, the analytical data is similar to measured data from a dielectric rod antenna GPR system. Soil modification through an optimal homogeneous soil water content is shown to improve target detection, but optimal water contents decrease when the target depths increase since loss even in the lower water content soil will become significant for very deep targets. In many situations, it may not be possible to obtain effective soil modification using water alone. Soil modification through the addition of water and liquid nitrogen 
was also described. Simulated results from the PO model confirm that an "optimal" amount of liquid nitrogen should exist to enhance target visibility. Initial measurements with a dielectric rod antenna GPR system also demonstrate that the addition of liquid nitrogen to excessively wet soils can reduce loss and enhance target visibility. Results from this study show potential for soil modification techniques to improve target detection. Clearly the soil modification procedures described can produce improvement to microwave GPR systems in two limiting cases: targets with strong scattering responses (i.e., metallic) obscured by excessive loss in the background medium and targets whose dielectric constant is identical to that of the background medium. In addition, the soil modification techniques described can potentially produce advantages not only in target detection but also in target identification, since scattered target signatures should be enhanced under larger dielectric contrasts.

\section{REFERENCES}

[1] A. C. Dubey, J. F. Harvey, J. T. Broach, and R. E. Dugan, "Detection and remediation technologies for mines and minelike targets V," in Proc. SPIE, vol. 4038, 2000.

[2] J. T. Johnson, J. Jenwatanavet, N. Wang, R. Caldecott, and J. Young, "Waveguide studies of soil modification techniques for enhanced mine detection with ground penetrating radar," in Detection and Remediation Technologies for Mines and Minelike Targets III, Proc. SPIE, vol. 3392, A. C. Dubey, J. F. Harvey, and J. T. Broach, Eds., 1998, pp. 716-724.

[3] J. Jenwatanavet, J. T. Johnson, N. Wang, and R. Caldecott, "Development of soil modification techniques for enhanced detection/discrimination," in IGARSS'98 Conf. Proc., vol. 1, 1998, pp. 210-212.

[4] M. H. Powers and G. R. Olhoeft, "Computer modeling to transfer GPR UXO detectability knowledge between sites," in UXO Forum 1996, Conf. Proc., 1996, pp. 347-356.

[5] H. Frohlich, Theory of Dielectrics; Dielectric Constant and Dielectric Loss. New York, NY: Oxford Univ. Press, 1958.

[6] J. T. Johnson, J. Jenwatanavet, and N. Wang, "Soil modification studies for enhanced mine detection with ground penetrating radar," in Detection and Remediation Technologies for Mines and Minelike Targets IV, Proc. SPIE, vol. 3710, A. C. Dubey, J. F. Harvey, J. T. Broach, and R. E. Dugan, Eds., 1999, pp. 739-748.

[7] N. Geng, D. Jackson, and L. Carin, "On the resonances of a dielectric BOR buried in a dispersive layered medium," IEEE Trans. Antennas Propagat., vol. 47, pp. 1305-1313, Aug. 1999

[8] N. Geng and L. Carin, "Wide-band electromagnetic scattering from a dielectric BOR buried in a layered lossy dispersive medium," IEEE Trans. Antennas Propagat., vol. 47, pp. 610-619, Apr. 1999.

[9] T. Dogaru and L. Carin, "Time-domain sensing of targets buried under a rough air-ground interface," IEEE Trans. Antennas Propagat., vol. 46, pp. 360-372, Mar. 1998.
[10] N. Geng and L. Carin, "Short-pulse electromagnetic scattering from arbitrarily oriented subsurface ordnance," IEEE Trans. Geosci. Remote Sensing, vol. 37, pp. 2111-2113, July 1999.

[11] N. Geng, C. Baum, and L. Carin, "On the low-frequency natural response of conducting and permeable targets," IEEE Trans. Geosci. Remote Sensing, vol. 37, pp. 347-359, Jan. 1999.

[12] E. Newman, "A User's Manual for the Electromagnetic Surface Patch Code: Preliminary Version ESP5.0," ElectroScience Laboratory, The Ohio State University, unpublished report, 1997.

[13] S. Nag, J. L. Peters, I. J. Gupta, and C.-C. Chen, "Ramp response for the detection of anti-personnel mines," in Detection and Remediation Technologies for Mines and Minelike Targets IV, Proc. SPIE, vol. 3710, A. C. Dubey, J. F. Harvey, J. T. Broach, and R. E. Dugan, Eds., 1999, pp. 1313-1322.

[14] A. V. der Merwe and I. J. Gupta, "A novel signal processing technique for clutter reduction in GPR measurements of small, shallow land mines," IEEE Trans. Geosci. Remote Sensing, vol. 38, pp. 2627-2637, 2000

[15] F. Ulaby, R. Moore, and A. Fung, Microwave Remote Sensing: Active and Passive. New York: Addison-Wesley, 1981, vol. 3.

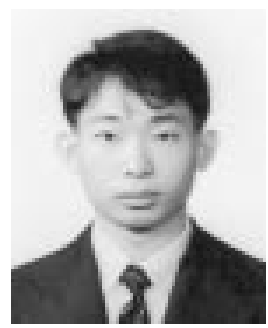

Jatupum Jenwatanavet obtained the B.E. degree in electrical engineering from Chulalongkorn University, Bangkok, Thailand, and the M.S. degree in electrical engineering from The Ohio State University, Columbus. From 1997 to 1999, he was a graduate research associate at The ElectroScience Laboratory, The Ohio State University. His research interests include theoretical and experimental studies in remote sensing, radar, scattering, and antennas. He is currently an electrical engineer with Motorola, Inc. in Piscataway, NJ.

Joel T. Johnson (M'96) received the B.E.E. degree in electrical engineering from the Georgia Institute of Technology in 1991 and the S.M. and Ph.D. degrees from the Massachusetts Institute of Technology in 1993 and 1996, respectively.

He is currently an Associate Professor in the Department of Electrical Engineering and ElectroScience Laboratory of The Ohio State University. His research interests are in the areas of microwave remote sensing, propagation, and electromagnetic wave theory.

Dr. Johnson is an associate member of commissions B and F of the International Union of Radio Science (URSI), and a member of Tau Beta Pi, Eta Kappa $\mathrm{Nu}$, and Phi Kappa Phi. He received the 1993 best paper award from the IEEE Geoscience and Remote Sensing Society, and was named an Office of Naval Research Young Investigator, National Science Foundation Career awardee, and PECASE award recipient in 1997. 